\title{
Arterial Spin Labeling MRI for Quantitative Assessment of Cerebral Perfusion Before and After Cerebral Revascularization in Children with Moyamoya Disease
}

\author{
Ji Young $\mathrm{Ha}, \mathrm{MD}, \mathrm{PhD}^{1}$, Young Hun Choi, $\mathrm{MD}^{2,3}$, Seunghyun Lee, $\mathrm{MD}^{2}$, Yeon Jin Cho, $\mathrm{MD}^{2,3}$, \\ Jung-Eun Cheon, $M D$, PhD ${ }^{2,3,4}$, In-One Kim, MD, PhD',3,4, Woo Sun Kim, MD, PhD ${ }^{2,3,4}$
}

${ }^{1}$ Department of Radiology, Gyeongsang National University Changwon Hospital, Changwon, Korea; ${ }^{2}$ Department of Radiology, Seoul National University Hospital, Seoul, Korea; ${ }^{3}$ Department of Radiology, Seoul National University College of Medicine, Seoul, Korea; ${ }^{4}$ Institute of Radiation Medicine, Seoul National University Medical Research Center, Seoul, Korea

Objective: To determine the correlation between cerebral blood flow (CBF) on arterial spin labeling (ASL) MRI and the degree of postoperative revascularization assessed on digital subtraction angiography in children with moyamoya disease (MMD). Materials and Methods: Twenty-one children (9 boys and 12 girls; mean age, $8.4 \pm 3.6$ years; age range, 3-16 years) with MMD who underwent both pseudocontinuous ASL MRI at 1.5T and catheter angiography before and after superficial temporal artery encephaloduroarteriosynangiosis were included in this retrospective study. The degree of revascularization in the middle cerebral artery (MCA) territory was evaluated on external carotid angiography and was graded on a 3-point scale. On ASL CBF maps, regions of interest were manually drawn over the MCA territory of the operated side at the level of the centrum semi-ovale and over the cerebellum. The normalized CBF (nCBF) was calculated by dividing the CBF of the MCA territory by the CBF of the cerebellum. Changes in $\mathrm{nCBFs}$ were calculated by subtracting the preoperative nCBF values from the postoperative $\mathrm{nCBF}$ values. The correlation between $\mathrm{nCBF}$ changes measured with ASL and the revascularization grade from direct angiography was evaluated.

Results: The $\mathrm{nCBF}$ value on the operated side increased after the operation $(p=0.001)$. The higher the degree of revascularization, the greater the $\mathrm{nCBF}$ change was: poor revascularization (grade 1$),-0.043 \pm 0.212$; fair revascularization (grade 2), $0.345 \pm 0.176$; good revascularization (grade 3), $0.453 \pm 0.182(p=0.005$, Jockheere-Terpstra test). The interobserver agreement was excellent for the measured CBF values of the three readers (0.91-0.97).

Conclusion: The $\mathrm{nCBF}$ values of the MCA territory obtained from ASL MRI increased after the revascularization procedure in children with MMD, and the degree of $\mathrm{nCBF}$ change showed a significant correlation with the degree of collateral formation evaluated via catheter angiography.

Keywords: Moyamoya; Arterial spin labeling; Cerebral blood flow; Cerebral perfusion; Pediatric; Angiography

\section{INTRODUCTION}

Moyamoya disease (MMD), which is relatively common in Asia, is an idiopathic cerebrovascular disease that is characterized by progressive steno-occlusive changes at the terminal portion of the internal carotid arteries (ICAs) and its main branches with the compensatory development of a fine vascular network, termed moyamoya vessels (1, 2). During the progressive occlusion of the bilateral ICAs, the leptomeningeal collateral vessels from the posterior circulation, and the transdural collateral vessels from the external carotid artery, the moyamoya vessels may develop

Received September 20, 2018; accepted after revision March 13, 2019.

Corresponding author: Young Hun Choi, MD, Department of Radiology, Seoul National University Hospital, 101 Daehak-ro, Jongno-gu, Seoul 03080, Korea.

- Tel: (822) 2072-3608 • Fax: (822) 747-5781 • E-mail: choiyounghun@gmail.com

This is an Open Access article distributed under the terms of the Creative Commons Attribution Non-Commercial License (https:// creativecommons.org/licenses/by-nc/4.0) which permits unrestricted non-commercial use, distribution, and reproduction in any medium, provided the original work is properly cited. 
to supply the ischemic brain $(3,4)$. During this change in cerebral hemodynamics, transient ischemic attacks or cerebral infarction can occur, leading to major neurologic and cognitive impairments (1).

Surgical revascularization surgery is considered the standard treatment to improve cerebral hemodynamics and reduce the risk of a subsequent stroke. Indirect bypass surgery that induces spontaneous angiogenesis between the brain surface and the vascularized donor tissues is technically simple to do and has been widely used especially in pediatric patients $(1,5,6)$. Additionally, bifrontal encephalogaleo (periosteal) synangiosis $(E G(P)$ $S)$ can be performed to reinforce the anterior circulation in patients with frontal lobar hypoperfusion $(7,8)$.

Evaluation of cerebral hemodynamics after cerebral revascularization is clinically meaningful for the assessment of the therapeutic effect and prognosis. There are several ways to evaluate the postsurgical revascularization status in moyamoya patients. Digital subtraction angiography (DSA) is still the gold standard for assessing the development of collateral pathways through direct or indirect bypass surgery $(3,9)$. However, DSA is invasive and time-consuming, especially in pediatric patients and may cause procedurerelated complications.

Dynamic susceptibility contrast (DSC) perfusion MRI has been used to noninvasively evaluate cerebral hemodynamics in association with ischemic brain lesions. Several reports demonstrated that DSC MRI can allow for the detection of hemodynamic changes after revascularization surgery $(4,10)$. However, DSC perfusion MRI requires gadolinium as a contrast agent and with increasing concerns about gadolinium deposition in the brain, it is recommended that DSC perfusion MRI be used with caution $(11,12)$.

CT perfusion scanning may be another option because of its high accessibility and short examination times, but exposure to radiation is unavoidable and the radiation dose is increased because of the repeated scans, which is especially concerning in children (13).

Recently, arterial spin labeling (ASL) MRI, which is based on endogenous arterial blood protons as a freely diffusible tracer, is a promising noninvasive perfusion imaging method for assessment of cerebral blood flow (CBF) without administration of contrast agents and without exposing children to radiation. Perfusion measurement using ASL has been increasingly applied in several imaging studies (14, 15). In MMD, there have been several studies for evaluation of the hemodynamic status and postoperative collateral vessels via ASL perfusion MRI (16-20). However, to the best of our knowledge, the changes in CBF values measured via ASL with DSA-assessed revascularization degree after indirect neovascularization surgery in pediatric patients have not yet been reported. Therefore, the purpose of this study was to assess the role of ASL MRI in quantitatively evaluating changes in CBF after indirect revascularization in children with MMD.

\section{MATERIALS AND METHODS}

Our Institutional Review Board approved this study. Informed consent requirement was waived due to the study's retrospective nature.

\section{Patients}

From June 2016 to March 2017, we retrospectively reviewed children with MMD at our institution. The inclusion criteria were 1) patients, younger than 18 years, diagnosed with MMD, 2) consecutive patients who underwent both ASL perfusion MRI and DSA imaging before and after the firststage indirect revascularization operation on the severe side, and 3) short time interval within 15 days between ASL perfusion MRI and DSA imaging before and after surgery. A total of 21 consecutive children ( 9 boys and 12 girls; mean age, $8.4 \pm 3.6$ years; age range, 3-16 years) were included in this study.

After the initial diagnosis of MMD, patients usually underwent two stages of indirect revascularization of the brain areas with vascular insufficiency. Usually, the operations consisted of bilateral encephaloduroarteriosynangiosis (EDAS) using the superficial temporal artery (STA) for the middle cerebral artery $(M C A)$ territory and bifrontal $\mathrm{EG}(\mathrm{P}) \mathrm{S}$ for the anterior cerebral artery territory, as previously described $(7,8,21)$. The imaging protocol for MMD patients at our institution was as follows: 1) Baseline MRI including MRI angiography, MRI perfusion using both DSC imaging, and ASL imaging and DSA before surgery for diagnosis of MMD and preoperative evaluation of vascular anatomy. 2) Postoperative MRI perfusion including DSC and ASL MRI and DSA within one to three months after the first-stage operation of unilateral indirect revascularization on the severe side. 3) Second postoperative follow-up MRI including MRI angiography, DSC, and ASL perfusion MRI within six months after the second-stage surgery for the contralateral side. 


\section{ASL Perfusion MRI}

ASL perfusion MRI was performed using a 1.5T MRI system (MAGNETOM Avanto, Siemens Healthineers, Erlangen, Germany) with a 12-channel head coil. Pseudocontinuous arterial spin labeling (pCASL) scans with two-dimensional single-shot-gradient-echo echo-planar imaging readout were performed using a labeling pulse duration of 1.8 seconds (Number of RF blocks of 98) and a post-labeling delay (PLD) of 1.5 seconds. Label offset from the center of the imaging region was $90 \mathrm{~mm}$ and the number of measurements was 60 (30 pairs of control and tag images). Other scan parameters were repetition time/echo time 4290/22 ms, $1578 \mathrm{~Hz} /$ pixel receiver bandwidth, $10 \mathrm{~mm}$ slice thickness, ten slices, 25 $\times 25 \mathrm{~cm}$ field of view, $96 \times 96$ matrix, and a generalized autocalibrating partially parallel acquisition acceleration factor of 2. The total scan time was 4.5 minutes.

The quantification model for pCASL technique has been previously reported $(22,23)$. The postprocessing was performed using SPM8 (Wellcome Trust Center for NeuroImaging, University College London, London, UK), ASL toolbox (24) and MATLAB (Mathworks, Natick, MA, USA) scripts. The calculation of CBF values uses the following equation:

$$
r C B F=\frac{\lambda \cdot \Delta M \cdot R_{1 a}}{2 \cdot \alpha \cdot M_{0}\left(e^{-w \cdot R_{1 a}}-e^{-(\tau+w) \cdot R_{1 a}}\right)}
$$

where $\mathrm{rCBF}$ is the relative CBF; $\lambda$ is the blood and tissue water partition coefficient $(0.9 \mathrm{~mL} / \mathrm{g}) ; \Delta \mathrm{M}$ is the difference in signal intensity between control image and labeling image; $R_{1 \mathrm{a}}$ is the longitudinal relaxation rate of blood $(0.719$ seconds $^{-1}$ at $\left.1.5 \mathrm{~T}\right) ; \alpha$ is the labeling efficiency $(0.85) ; M_{0}$ is the equilibrium magnetization of brain (control image intensity); $w$ is the PLD time; $\tau$ is the duration of the labeling pulse (18.4 msec * Number of RF blocks).

\section{DSA}

Direct catheter cerebral angiography was performed in a dual-plane angiographic machine (Axiom Artis; Siemens Healthineers) by four pediatric radiologists with 5, 5, 6, and 13 years of experience, respectively. A $4 \mathrm{~F}$ or $5 \mathrm{~F}$ Davis angiographic catheter (A\&A Medical Device, Seongnam, Korea) was used according to the patient's femoral artery diameter. Conventional DSA images included anteroposterior and lateral projections with selective injection of the respective internal carotid, the external carotid, and the vertebral artery. Low-osmolality iodinated contrast media (iopamidol; Pamiray 250; Dongkuk Pharm., Seoul, Korea) was administered using an automated injector (Angiomat Illumena; Liebel-Flarsheim, Cincinnati, $\mathrm{OH}$, USA). The injection rate and amount were determined by the operator based on the size of each vessel.

\section{Quantitative Analysis of CBF on ASL}

From the ASL maps obtained before and after the first indirect revascularization operation, three independent and blinded board-certified pediatric radiologists (R1, R2, and R3 with 5, 5, and 6 years of clinical experience, respectively, independently and manually drew regions of interest (ROIs) over the bilateral MCA territories as large as possible on the ASL maps at the level of the centrum semi-ovale (Fig. 1). Before the analysis, the three readers reviewed five cases that were not included in this study group.

The ROI of the MCA territory at the level of the centrum semi-ovale was defined as the cortex and the subcortical white matter supplied from the leptomeningeal arteries of the MCA. The ROI excluded the deep white matter of the centrum semi-ovale, the watershed zone between the superficial territories of the MCA, and the anterior cerebral artery (25). The obtained mean signal intensity value of each ROI represented the absolute CBF value in the MCA territory $\left(\mathrm{CBF}_{M C A}\right)(16)$. All values of $\mathrm{CBF}$ maps were measured in absolute units $(\mathrm{mL} / 100 \mathrm{~g} / \mathrm{min})$.

For CBF normalization, additional ROIs were drawn on the right and left cerebellar hemispheres, respectively. The absolute $\mathrm{CBF}$ values of cerebellum $\left(\mathrm{CBF}_{\mathrm{Cbll}}\right)$ was defined as the mean of right and left $\mathrm{CBF}_{\mathrm{cbll}}$. The size of the ROI was set to be at least $2000 \mathrm{~mm}^{2}$ in the MCA territory of each cerebral hemisphere and $350 \mathrm{~mm}^{2}$ in the cerebellum.

Normalized $\mathrm{CBF}$ ( $\mathrm{nCBF}$ ) was calculated using the following equation: $n C B F=C B F_{M C A} / C B F_{C b l} \cdot n C B F$ values were obtained from both operated side and contralateral sides on both pre- and postoperative ASL images. To evaluate the change in the $\mathrm{nCBF}$ value on the operated side after surgery, $\triangle \mathrm{nCBF}$ was calculated as $\triangle \mathrm{nCBF}=$ postoperative $\mathrm{nCBF}$ preoperative $\mathrm{nCBF}$.

\section{Grading of Postoperative Revascularization in the MCA Territory on DSA}

For visual grading of the revascularization degree after surgery, two board-certified pediatric radiologists (with 5 years and 13 years of experience, respectively) independently reviewed the DSA images and the results were determined consensually. The findings of ASL image 


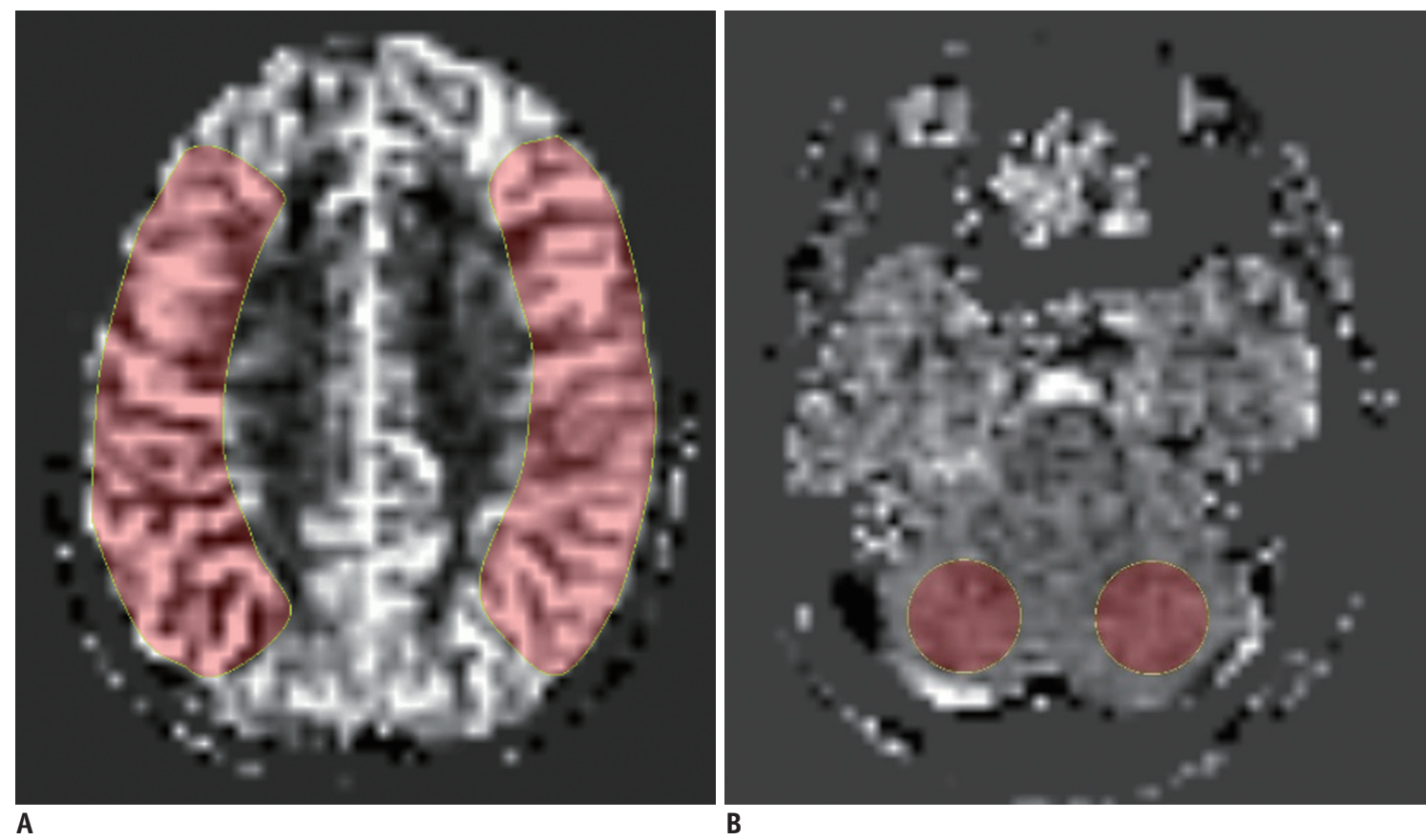

Fig. 1. Imaging example of ROI placement for CBF measurements in ASL images.

A. ROIs created manually over MCA territory along level of centrum semi-ovale on operated side and contralateral side. B. Additional ROIs drawn in cerebellum for normalization on right and left cerebellar hemispheres. Absolute $\mathrm{CBF}_{\mathrm{cbll}}$ was defined as mean of right and left $C B F_{\mathrm{cbll}}$. $\mathrm{ASL}=$ arterial spin labeling, $\mathrm{CBF}=$ cerebral blood flow, $\mathrm{CBF}_{\mathrm{cbll}}=\mathrm{CBF}$ of cerebellum, $\mathrm{MCA}=$ middle cerebral artery, $\mathrm{ROI}=$ region of interest

were blinded when two radiologists reviewed DSA images. The transdural collaterals at the synangiosis site of the MCA territory were graded according to the criteria proposed by Matsushima et al. (6) for the evaluation of transdural collaterals after EDAS (26).

Visual assessment of the perfused area by neovascularization after surgery on external carotid artery angiography was scored on a 3-point scale as follows: 1 , poor (area perfused by the synangiosis is less than $1 / 3$ of the MCA territory); 2 , fair (area perfused by the synangiosis is between $1 / 3$ and $2 / 3$ of the MCA territory); 3, good (area perfused by the synangiosis is greater than $2 / 3$ of the MCA territory).

\section{Statistical Analysis}

For each parameter, the normality of data was assessed with the Kolmogorov-Smirnov test. Absolute CBFs, nCBFs, and the areas of ROI were compared between pre- and postoperative images using a paired $t$ test. The change in nCBF after surgery according to the DSA revascularization grade was compared using one-way ANOVA with post-hoc Tukey Honestly Significant Difference test. The JockheereTerpstra test was performed for trend analysis of the change in $\mathrm{nCBF}$ according to the neovascularization degree. All statistical analyses were performed using SPSS v. 22.0 statistical software (IBM Corp., New York, NY, USA) and
MedCalc software v. 12.1.0 (MedCalc Software, Mariakerke, Belgium). The interobserver variability for CBF measurement of both MCA territory and cerebellum from the three readers was analyzed by calculating the intraclass correlation coefficient (ICC: $0.00-0.20$, poor agreement; 0.21-0.40, fair; $0.41-0.60$, moderate; $0.61-0.80$, good; and $0.81-1.00$, excellent).

\section{RESULTS}

\section{Patients}

A summary of the patient characteristics is shown in Table 1. Twenty patients were diagnosed with typical bilateral MMD and one patient had unilateral MMD. As an initial indirect operation, 13 of 21 patients received EDAS only, and eight patients underwent EDAS with EG(P)S.

The mean period between pre-operative ASL and DSA imaging was $2.7 \pm 4.6$ days (range, $0-15$ days). The mean period between pre-operative ASL and surgery was $4.1 \pm$ 5.1 days (range, 1-22 days). The mean period between surgery and postoperative ASL, and between surgery and postoperative DSA was $77.1 \pm 43.4$ days (range, 34-245 days) and $77.6 \pm 43.3$ days (range, 34-245 days), respectively. The interval between postoperative ASL and DSA imaging was $0.5 \pm 0.5$ days (range, $0-1$ day). 
Table 1. Clinical Data and Imaging Findings

\begin{tabular}{|c|c|}
\hline Category & No. of Patients \\
\hline Mean age (range) & $8.4 \pm 3.6$ years ( $3-16$ years) \\
\hline Sex (male:female) & $9: 12$ \\
\hline Ethnic origin & Asian (Korean) \\
\hline Underlying disease* & None \\
\hline Family history & 1/21 (grandmother; MMD) \\
\hline \multicolumn{2}{|l|}{ Clinical symptom (\%) } \\
\hline TIA & $16 / 21(76.2)$ \\
\hline Headache & $6 / 21(28.6)$ \\
\hline Involuntary movement & $1 / 21(4.8)$ \\
\hline Seizure & $2 / 21(9.5)$ \\
\hline Persistent weakness & $2 / 21(9.5)$ \\
\hline \multicolumn{2}{|l|}{ Lesion on MRI (\%) } \\
\hline None & $12 / 21(57.1)$ \\
\hline Infarction & $6 / 21(28.6)$ \\
\hline ICH or IVH & $2 / 21(9.5)$ \\
\hline Cerebral hemi-atrophy & $1 / 21(4.8)$ \\
\hline PCA involvement & $6 / 21$ \\
\hline Surgery side (right:left) & $11: 10$ \\
\hline \multicolumn{2}{|l|}{ Initial surgery } \\
\hline EDAS only & $13 / 21$ \\
\hline EDAS with $\mathrm{EG}(\mathrm{P}) \mathrm{S}$ & $8 / 21$ \\
\hline \multicolumn{2}{|l|}{ Postop. outcome } \\
\hline Postop. infarction & $4 / 21$ \\
\hline Postop. SDH & $1 / 21$; second surgery needed \\
\hline
\end{tabular}

*Underlying disease such as neurofibromatosis type 1 and Down syndrome, etc. EDAS = encephaloduroarteriosynangiosis, EG(P) $\mathrm{S}=$ encephalogaleo (periosteal) synangiosis, $\mathrm{ICH}=$ intracerebral hematoma, IVH = intraventricular hemorrhage, MMD = moyamoya disease, $\mathrm{PCA}=$ posterior cerebral artery, Postop. $=$ postoperative, $\mathrm{SDH}=$ subdural hemorrhage, $\mathrm{TIA}=$ transient ischemic attack

\section{CBF Changes after Cerebral Revascularization on ASL}

Mean absolute CBF values on the operated side by all readers at pre- and postoperative were $80.26 \pm 31.2$ (range, $12.9-150.4 \mathrm{~mL} / 100 \mathrm{~g} / \mathrm{min}$ ) and $89.39 \pm 23.6$ (range, 20.6$136.1 \mathrm{~mL} / 100 \mathrm{~g} / \mathrm{min}$ ), respectively. Mean absolute CBF values on the contralateral side at pre- and postoperative were $88.69 \pm 34.0$ (range, $14.5-146.5 \mathrm{~mL} / 100 \mathrm{~g} /$ $\min$ ) and $80.59 \pm 30.5$ (range, $10.9-142.8 \mathrm{~mL} / 100 \mathrm{~g} /$ $\mathrm{min})$, respectively. And the absolute $\mathrm{CBF}_{\mathrm{cbll}}$ at pre- and postoperative were $52.14 \pm 15.2$ (range, 31.0-118.9 $\mathrm{mL} / 100 \mathrm{~g} / \mathrm{min}$ ) and $50.38 \pm 9.3$ (range, $34.2-69.7 \mathrm{~mL} / 100$ $\mathrm{g} / \mathrm{min})$, respectively.

After revascularization surgery, there was no significant increase of absolute CBF values measured on the operated side by R2 and R3 (preoperative vs. postoperative; $80.66-80.78$ vs. $88.19-89.98 \mathrm{~mL} / 100 \mathrm{~g} / \mathrm{min}, p=0.070$ 0.115). The absolute CBF on the operated side by R1 was significantly increased (preoperative vs. postoperative; 79.33 vs. $89.99 \mathrm{~mL} / 100 \mathrm{~g} / \mathrm{min}, p=0.020$ ). Absolute CBF values at the contralateral side were not significantly different before and after surgery in all readers (preoperative vs. postoperative; $88.02-89.29$ vs. $80.02-81.05 \mathrm{~mL} / 100$ $\mathrm{g} / \mathrm{min}, p=0.118-0.145)$. The $\mathrm{CBF}_{\mathrm{cbll}}$ were also not significantly different in all readers (preoperative vs. postoperative; $52.04-52.62$ vs. $48.30-48.96 \mathrm{~mL} / 100 \mathrm{~g} / \mathrm{min}$, $p=0.123-0.178)$.

nCBF values calculated on the operated side in all readers were significantly increased after surgery (preoperative vs. postoperative; $1.53-1.57$ vs. $1.84-1.89, p=0.001$ ). $0 n$ the contralateral side, there was no difference between nCBF values before and after surgery preoperative vs. postoperative; $1.73-1.77$ vs. $1.69-1.73, p=0.651-0.944$ ) (Table 2).

\section{Comparison between the Revascularization Degree on DSA and CBF Changes on ASL}

Revascularization degree was interpreted as grade 1 in four patients $(19.0 \%)$, grade 2 in seven patients $(33.3 \%)$, and grade 3 in the remaining ten patients $(47.6 \%)$ on DSA. In three readers, the change in nCBF after surgery $(\triangle \mathrm{nCBF})$ was calculated for each group, respectively. $O \mathrm{n}$ the operated side, the mean of $\triangle \mathrm{nCBF}$ in these three groups showed a significant difference in all readers $(p=0.003-0.005)$. The average value of $\triangle \mathrm{nCBF}$ in three readers showed a significant difference between the three groups $(p=0.001)$. In the post-hoc analysis for the averaged $\triangle \mathrm{nCBF}$, there was no significant difference between group 2 and $3(p=0.448)$, but there was a significant difference between group 1 and $2(p=0.010)$ and group 1 and $3(p=0.001)$ (Fig. 2, Table 3$)$. The trend analysis showed that the higher revascularization grade, the greater the mean of $\triangle \mathrm{nCBF}(p=0.005)$.

Representative images are shown in Figures 3 and 4.

\section{Interobserver Agreement}

The interobserver agreement on CBF values measured by three readers in bilateral MCA territories and cerebellum are shown in Table 4. All the calculated interobserver agreement were excellent between the three readers (ICC, 0.91-0.97).

\section{DISCUSSION}

This study assessed whether CBF changes measured by ASL MRI can predict the degree of revascularization after 
Table 2. Comparison between Pre- and Postoperative CBF Values in Patients $(n=21)$ Who Underwent Unilateral Revascularization Based on ASL Measurements for Each Reader

\begin{tabular}{|c|c|c|c|c|}
\hline Reader & Parameter & Preoperative ASL & Postoperative ASL & $P$ \\
\hline \multirow{7}{*}{ R1 } & Absolute CBF* & & & \\
\hline & Operated side & $79.33 \pm 31.15$ & $89.99 \pm 25.48$ & 0.020 \\
\hline & Contralateral side & $88.02 \pm 33.92$ & $80.72 \pm 29.88$ & 0.145 \\
\hline & Cerebellum $^{\dagger}$ & $52.62 \pm 12.53$ & $48.51 \pm 9.58$ & 0.123 \\
\hline & $\mathrm{nCBF}^{\ddagger}$ & & & \\
\hline & Operated side & $1.53 \pm 0.53$ & $1.89 \pm 0.49$ & 0.001 \\
\hline & Contralateral side & $1.73 \pm 0.73$ & $1.72 \pm 0.67$ & 0.944 \\
\hline \multirow{7}{*}{ R2 } & Absolute CBF & & & \\
\hline & Operated side & $80.66 \pm 31.18$ & $88.19 \pm 22.16$ & 0.115 \\
\hline & Contralateral side & $88.76 \pm 34.48$ & $80.02 \pm 32.02$ & 0.118 \\
\hline & Cerebellum & $52.65 \pm 13.04$ & $48.96 \pm 9.44$ & 0.178 \\
\hline & $\mathrm{nCBF}$ & & & \\
\hline & Operated side & $1.55 \pm 0.51$ & $1.84 \pm 0.46$ & 0.001 \\
\hline & Contralateral side & $1.74 \pm 0.73$ & $1.69 \pm 0.71$ & 0.651 \\
\hline \multirow{7}{*}{ R3 } & Absolute CBF & & & \\
\hline & Operated side & $80.78 \pm 32.78$ & $89.98 \pm 24.28$ & 0.070 \\
\hline & Contralateral side & $89.29 \pm 35.24$ & $81.05 \pm 30.88$ & 0.124 \\
\hline & Cerebellum & $52.04 \pm 12.13$ & $48.30 \pm 8.74$ & 0.125 \\
\hline & $\mathrm{nCBF}$ & & & \\
\hline & Operated side & $1.57 \pm 0.54$ & $1.89 \pm 0.49$ & 0.001 \\
\hline & Contralateral side & $1.77 \pm 0.75$ & $1.73 \pm 0.71$ & 0.702 \\
\hline
\end{tabular}

Values are mean \pm standard deviation. Absolute or nCBF values were compared between pre- and postoperative ASL using paired $t$ test. ${ }^{*}$ Data are in units of $\mathrm{mL}$ per $100 \mathrm{~g}$ per minute, ${ }^{\dagger} \mathrm{Abs}$ olute $\mathrm{CBF}_{\mathrm{Cbu}}$ was defined as mean of right and left $\mathrm{CBF}_{\mathrm{cbll}},{ }^{\ddagger} \mathrm{nCBF}$ values are calculated as follows: $\mathrm{CBF}_{\mathrm{MCA}} / \mathrm{CBF}_{\mathrm{Cbll}} \cdot \mathrm{ASL}=$ arterial spin labeling, $\mathrm{CBF}=$ cerebral blood flow, $\mathrm{CBF}_{\mathrm{cbll}}=\mathrm{CBF}$ of cerebellum, $\mathrm{CBF}_{\mathrm{MCA}}=\mathrm{CBF}_{\text {value in the }}$ $\mathrm{MCA}$ territory, $\mathrm{MCA}=$ middle cerebral artery, $\mathrm{nCBF}=$ normalized $\mathrm{CBF}$

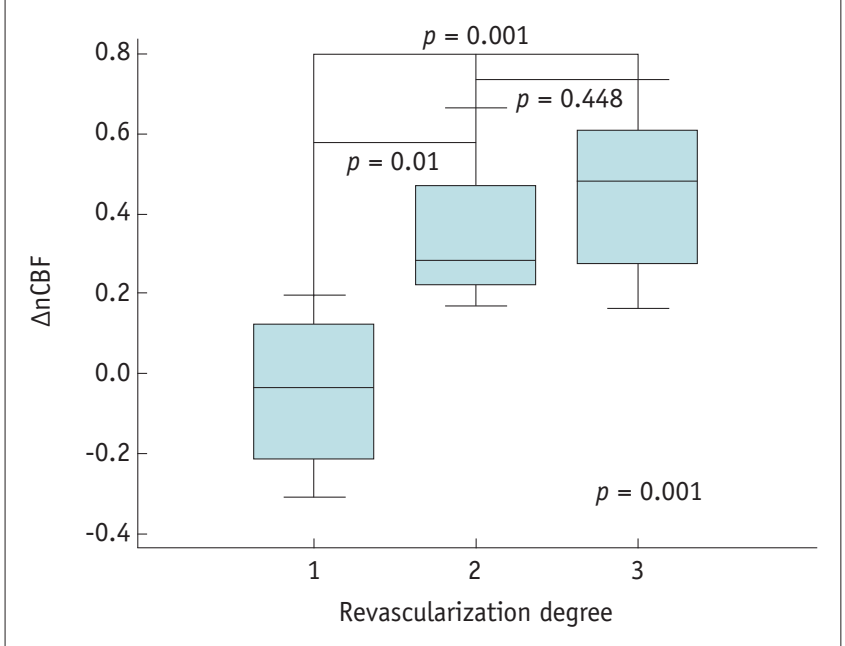

Fig. 2. Graph displaying $\mathrm{nCBF}$ changes measured with ASL according to angiographic revascularization degree (poor revascularization [grade 1], $-0.043 \pm 0.212$; fair revascularization [grade 2], $0.345 \pm 0.176 ;$ good revascularization [grade 3], $0.453 \pm$ $0.182[p=0.001]) . \mathrm{nCBF}=$ normalized $\mathrm{CBF}$

indirect revascularization surgery in children with MMD. Our findings demonstrated that $\mathrm{nCBF}$ values obtained from ASL increased after indirect revascularization surgery and reflected the degree of collateral formation evaluated on DSA. Therefore, ASL MRI could be used as a surrogate indicator of revascularization after operation in children with MMD.

Indirect revascularization is the most widely used surgical method in childhood MMD because of its simplicity. The optimal goal of STA EDAS is to reestablish CBF to the hypoperfused region using synangiosis from the parietal branches of the STA. After the revascularization surgery, artificially induced transdural collateral vessels develop at the flap site with concurrent and sequential progression of stenotic change in the ICA and diminishing of moyamoya vessels $(26,27)$. The neovascularization has been known to begin two weeks after the operation $(27,28)$. The goal of postoperative imaging would be to evaluate cerebral hemodynamic changes induced by a bypass surgery. For these purposes, several noninvasive imaging modalities have been performed such as DSC MRI, single-photon emission CT (SPECT), positron-emission tomography (PET) for evaluation of secondary hemodynamic changes, and MRI angiography for evaluation of the development of transdural 
Cerebral Perfusion Changes after Revascularization in Pediatric Moyamoya Disease

Table 3. Relationship between Angiographic Postoperative Revascularization Degree and Change in nCBF Value

\begin{tabular}{cccccc}
\hline Parameter & Reader & Grade 1 $(\mathrm{n}=4)^{*}$ & Grade 2 $(\mathrm{n}=7)$ & Grade 3 $(\mathrm{n}=10)$ & $P$ \\
\hline & $\mathrm{R} 1$ & $-0.054 \pm 0.302$ & $0.395 \pm 0.176$ & $0.495 \pm 0.256$ & 0.005 \\
$\Delta \mathrm{nCBF}^{\dagger}$ & $\mathrm{R} 2$ & $-0.039 \pm 0.248$ & $0.285 \pm 0.158$ & $0.416 \pm 0.182$ & 0.003 \\
& $\mathrm{R} 3$ & $-0.037 \pm 0.154$ & $0.356 \pm 0.262$ & $0.447 \pm 0.202$ & 0.005 \\
& Average $^{\ddagger}$ & $-0.043 \pm 0.212$ & $0.345 \pm 0.176$ & $0.453 \pm 0.182$ & 0.001 \\
& & & Group 1 and 2 & & 0.010 \\
Tukey HSD test & & & Group 2 and 3 & & 0.448 \\
& & & Group 1 and 3 & & 0.001 \\
\hline
\end{tabular}

Revascularization grading was evaluated via digital subtraction angiography imaging (baseline and postoperative follow-up image) in MCA territory. *Grade 1, poor (area perfused by synangiosis is less than $1 / 3$ of MCA territory); 2, fair (area perfused by synangiosis is between $1 / 3$ and $2 / 3$ of MCA territory); 3 , good (area perfused by synangiosis is greater than $2 / 3$ of MCA territory), ${ }^{\dagger}$ Change in $n C B F$ values is calculated as follows: $\triangle \mathrm{nCBF}=$ postoperative $n C B F$ - preoperative $n C B F$, ${ }^{\prime}$ Values averaged across three readers for $\triangle \mathrm{nCBF}$ values, ${ }^{\S}$ Post-hoc Tukey HSD test for averaged $\triangle \mathrm{nCBF}$ values across three readers. HSD = honestly significant difference

collateral vessels (1, 3, 4, 29-31). Recently, ASL MRI, which is based on endogenous blood as a freely diffusible tracer, has been suggested as a promising noninvasive perfusion imaging method for assessment of CBF without administration of contrast media and without radiation, which is of particular interest for pediatric patients.

Several studies examined the performance of ASL MRI for monitoring the hemodynamic status in $\operatorname{MMD}(16,18$ $20,32,33)$. CBF measurement using ASL MRI has shown good correlation with results obtained via PET (34) and DSC MRI (20) in patients with MMD. Several previous studies have demonstrated the improvement of perfusion after revascularization operation $(16,32,35)$. Sugino et al. (16) have reported that $\mathrm{nCBF}$ values from ASL were increased after direct revascularization in 14 pediatric and adult patients and it was comparable to SPECT (16). Recently, Lee et al. (36) reported that there was an excellent correlation between ASL MRI and DSA findings in CBF changes and anastomosis site patency in 145 adults with MMD who underwent direct revascularization. The number of studies in the pediatric population is limited. In a preliminary report by Blauwblomme et al. (35), CBFs measured with ASL MRI were increased after indirect revascularization in 15 pediatric patients $(6.8 \pm 3.4$ years, age range; $2.7-14$ years). While their study showed a simple improvement of CBF by comparing pre- and postoperative ASL values, our study demonstrated a significant positive correlation between the increase in nCBF and degree of revascularization.

In the present study, absolute CBF values measured on the operated side in all readers (R1/R2/R3) were slightly increased after surgery (mean difference, 10.66/7.53/9.19 $\mathrm{mL} / 100 \mathrm{~g}$ per minute). However, statistical significance was achieved only in R1 $(p=0.020)$. On the other hand,
$\mathrm{nCBF}$ values calculated on the operated side per cerebellum $\left(\mathrm{CBF}_{\mathrm{MCA}} / \mathrm{CBF}_{\mathrm{Cbl}}\right)$ increased after surgery with a statistically significant difference in all readers. There are two reasons that the difference in the absolute CBF values before and after surgery was smaller than in the previous study (35). The first is due to the size and location of ROI. In the prior study by Blauwblomme et al. (35), the authors drew a $10 \mathrm{~mm}$ ROI $\left(78.5 \mathrm{~mm}^{2}\right)$ in the dorsolateral cortex of the frontal lobe, corresponding to the area with a significant change of ASL CBF values in the preanalysis. This approach could have maximized the changes of ASL CBFs. However, identifying such areas with great CBF changes could be highly subjective and required preanalysis. In contrast, our study did not perform preanalysis and simply included a larger MCA territory (at least $2500 \mathrm{~mm}^{2}$ ) including cortex and subcortical white matter. We think that our approach of drawing large ROIs instead of local ROIs could be easier and clinically more practical and applicable, even though it might have underestimate absolute CBF changes. Since prior studies on quantitative CBF evaluation in MMD have included the cortex and subcortical white matter, and the actual change of CBF in nuclear image take place throughout brain parenchyma, we believe that our research including the cortex and subcortical white matter reflects the actual $\operatorname{CBF}(4,8,13,37)$. The second is due to the absolute CBF could be affected by a number of parameters such as blood T1, tissue T1 values and PLD. The delayed arterial transit time (ATT) in steno-occlusive disease such as MMD could have a significant effect on the ASL signal. Prolonged PLD in severe ATT delay could be helpful than normal children (14). In our study, PLD was 1500 ms, which is commonly used in studies of MMD $(20,36)$, longer than 1025 ms of Blauwblomme et al. (35). In the case of Blauwblomme et al. (35) with a short PLD, it is possible that the measured 

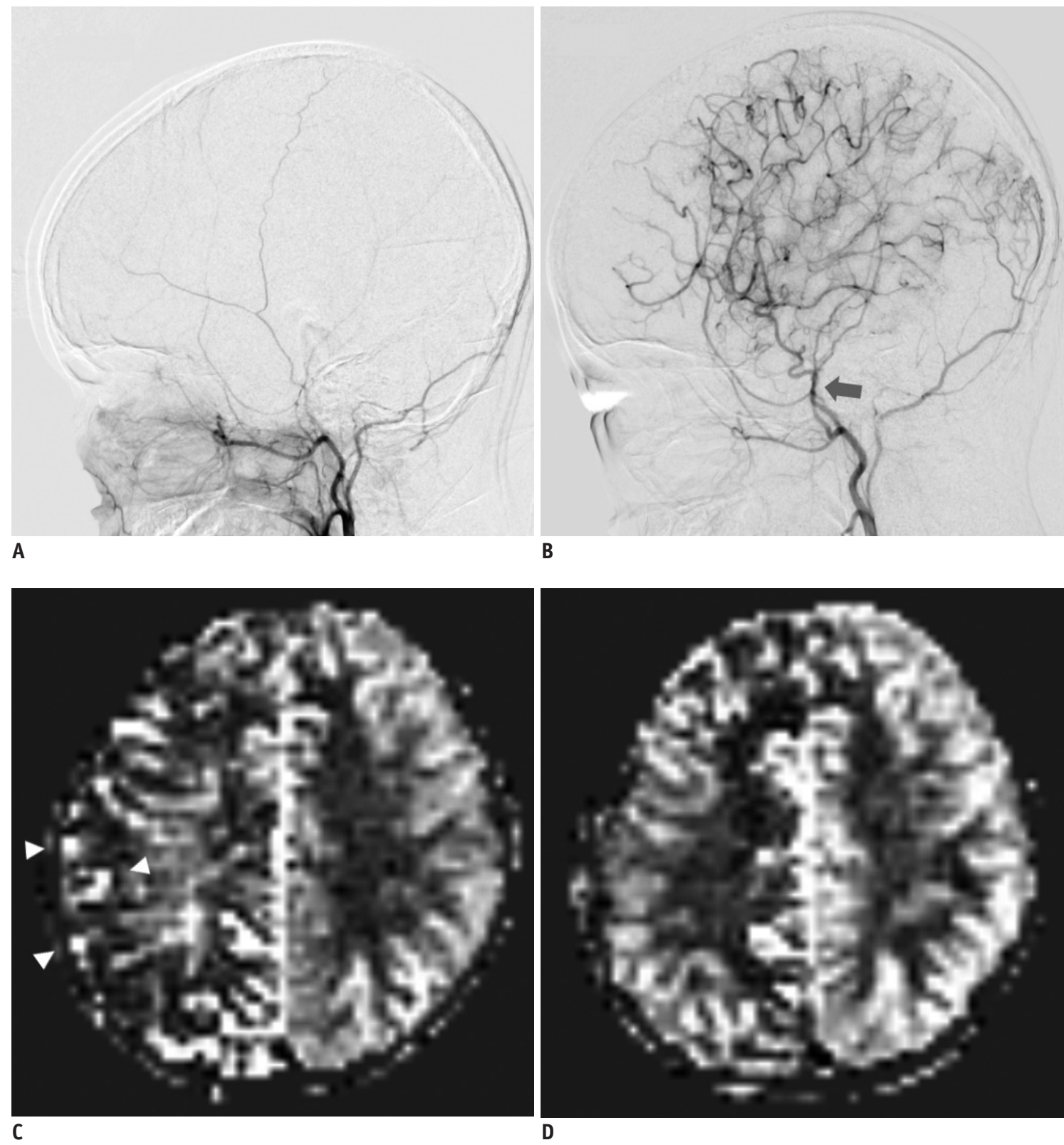

Fig. 3. Representative DSA and ASL images showing good postoperative outcome in 12-year-old girl with unilateral MMD who underwent right EDAS with right $\mathrm{EC}(\mathrm{P}) \mathrm{S}$.

A. Baseline DSA image showing lateral projection from right external carotid injections. B. Postoperative DSA image after surgery shows superficial temporal artery hypertrophy (arrow) and abundant collateral flow at greater than 2/3 of MCA territory; grade 3, good revascularization grade. C. Baseline ASL image shows decreased perfusion and ATA signals (arrowheads) reflecting late-arriving flow via collateral pathways. D. Postoperative ASL image shows increased perfusion with decreased ATA signals in right MCA territory, corresponding to DSA results. ATA = arterial transit artifact, DSA = digital subtraction angiography, $E C(P) S=$ encephalogaleo (periosteal) synangiosis, EDAS = encephaloduroarteriosynangios is, $M M D=$ moyamoya disease

preoperative ASL CBF was underestimated, thus the value of postoperative $\mathrm{CBF}$ minus preoperative $\mathrm{CBF}$ might be overestimated than the actual value. Our study would be more accurate because it is known that the ASL CBF values in the longer PLD are closer to true values in MMD.

In our study, the variation of the absolute CBF value was not consistent with the variation of $\mathrm{nCBF}$ value as it increased or decreased. The reason is the same as the reason why the $C B F$ value measured in $A S L$ are required to be normalized by the cerebellar CBF. The CBF value could vary widely in the same patient depending on the patient's perfusion status in MMD $(34,36)$. Similar to prior studies, our study also showed large variation in absolute CBF (e.g., $12.9-150.4 \mathrm{~mL} / 100 \mathrm{~g} / \mathrm{min}$ in the operated side 

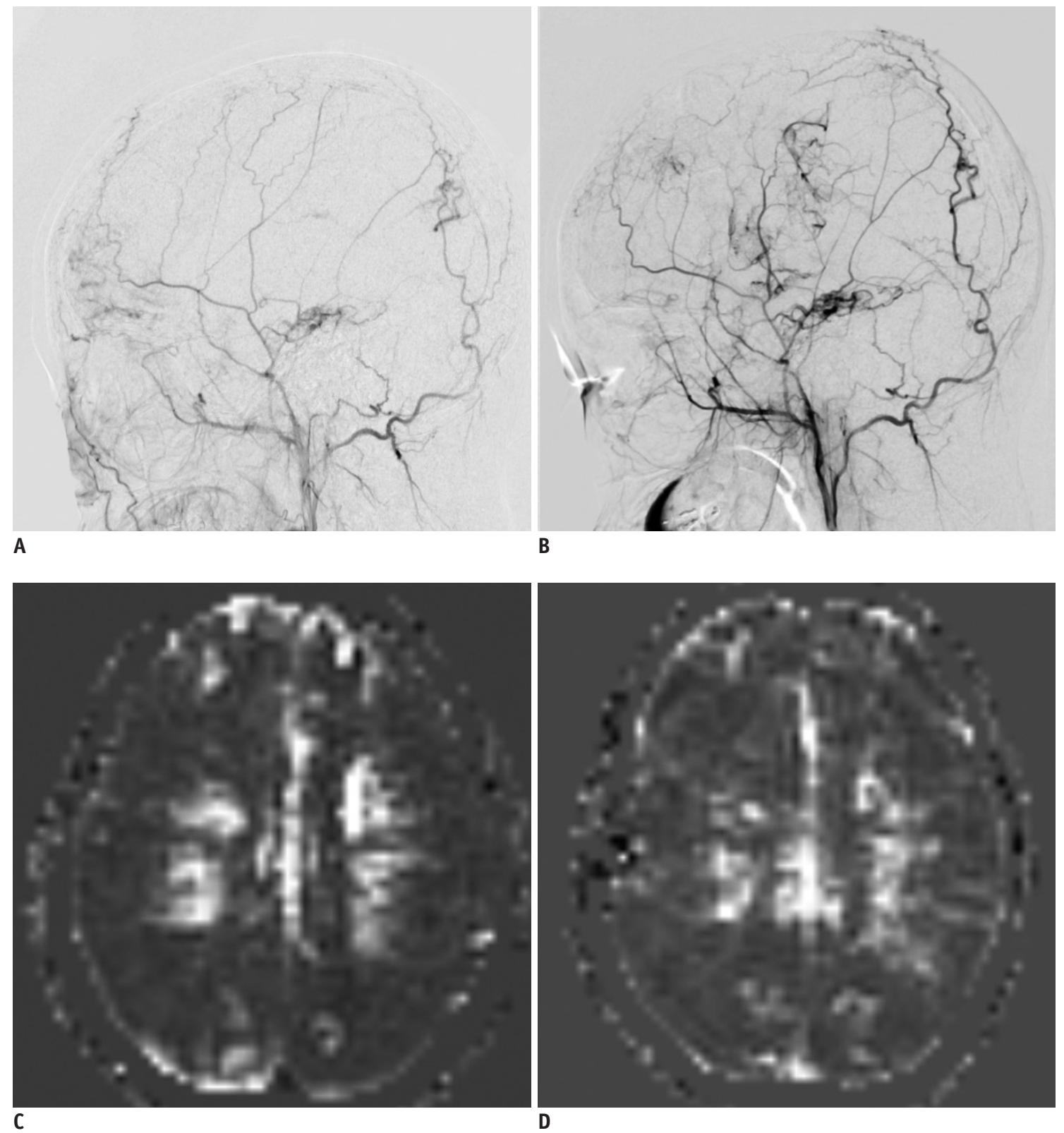

Fig. 4. Representative DSA and ASL images showing poor postoperative outcome in 16-year-old girl with bilateral MMD who underwent right EDAS with bifrontal EC(P)S.

A. Baseline DSA image showing lateral projection from right external carotid injections demonstrates preexisting transdural collaterals, indicating advanced stage of disease. B. Postoperative DSA image shows impaired collateral flow at less than $1 / 3$ of MCA territory; grade 1, poor revascularization grade. C. Baseline ASL image shows decreased perfusion in both MCA territories. D. Postoperative ASL image shows impaired perfusion, corresponding to DSA results.

at preoperative). To adjust for inter-subject variation and minimize the effect of transit-time error, we used nCBF values rather than absolute CBF values (36). There were two cases in which nCBF value was increased as a result of normalization, although absolute CBF value decreased. The increase in $\mathrm{nCBF}$ was consistent with DSA. E.g. In one patient with DSA grade 3; good, the absolute CBF decreased postoperatively (preoperative vs. postoperative; 71.5-74.4 vs. 59.1-63.2), but $\mathrm{nCBF}$ increased (preoperative vs. postoperative; $1.47-1.52$ vs. $1.71-1.86)$.

The primary benefits of ASL perfusion MRI are that it is non-invasive and radiation-free. Furthermore, no contrast agents are required which may cause adverse effects such as contrast-induced nephropathy of iodinated contrast media and the recently reported brain deposition of gadoliniumbased contrast agent. Therefore, ASL perfusion MRI is highly 
Table 4. Contingency Table for Interobserver Agreement of CBF Measured by Three Readers

\begin{tabular}{|c|c|c|}
\hline Parameter & Location & ICC $(95 \% \text { CI })^{*}$ \\
\hline \multirow{6}{*}{ Preoperative CBF } & MCA territory & \\
\hline & Right & $0.97(0.954-0.997)$ \\
\hline & Left & $0.94(0.887-0.976)$ \\
\hline & Cerebellum & \\
\hline & Right & $0.96(0.942-0.995)$ \\
\hline & Left & $0.95(0.910-0.983)$ \\
\hline \multirow{6}{*}{ Postoperative CBF } & MCA territory & \\
\hline & Right & $0.92(0.853-0.952)$ \\
\hline & Left & $0.96(0.942-0.998)$ \\
\hline & Cerebellum & \\
\hline & Right & $0.91(0.845-0.956)$ \\
\hline & Left & $0.94(0.871-0.977)$ \\
\hline
\end{tabular}

* ICC were calculated for interobserver agreement for three readers. $\mathrm{CI}=$ confidence interval, ICC $=$ intraclass correlation coefficient

recommended for children.

Our study has several limitations. First, a small number of patients was analyzed in this study. Second, we did not have a standard of reference such as PET, SPECT, or perfusion $\mathrm{CT}$ available to validate the quantitative $\mathrm{CBF}$ values on $\mathrm{ASL}$, because these techniques were not performed in our center. In several previous studies, collateral scoring on ASL perfusion MRI has shown good agreement with DSA in steno-occlusive disease $(17,38)$. It was thought that DSA was appropriate as a reference in our study, considering that the purpose of this study was to evaluate the developed collateral vessels. The third limitation concerns arterial transit artifact (ATA) issues. ATA is known to be a phenomenon in which late-arriving blood flow through collateral vessel appears as a strong ASL signal before it arrived at the brain tissue in ASL perfusion imaging $(16,39)$. This may affect the quantification of CBF values in patients with severe steno-occlusive disease such as MMD, in which collateral flow contributes significantly to cerebral perfusion. In this study, many ATAs might be included in large MCA ROIs and therefore, the bright signals of ATAs may have contributed to measuring CBF values higher than actual values, which could have made the difference in CBF values before and after surgery smaller. Fourth, interpretation of perfusion data in pediatric patients is complicated by age-related different perfusion change, PLD, blood T1, and tissue T1 values. In addition, depending on anesthesia or sedation for MRI, perfusion status could vary (40).

In conclusion, nCBFs measured by ASL MRI increased after indirect revascularization surgery in children with MMD and changes in $\mathrm{nCBF}$ perfusion values after revascularization surgery correlated with the degree of collateral formation evaluated via DSA. Our results suggest that ASL MRI can be used to monitor perfusion changes in pediatric patients with MMD after indirect revascularization.

\section{Conflicts of Interest}

The authors have no potential conflicts of interest to disclose.

\section{Acknowledgments}

The authors would like to thank Munyoung Paek for her advice regarding to technical background of the arterial spin labelling MRI into this work.

$$
\begin{aligned}
& \text { ORCID iDs } \\
& \text { Young Hun Choi } \\
& \text { https://orcid.org/0000-0002-1842-9062 } \\
& \text { Ji Young Ha } \\
& \text { https://orcid.org/0000-0001-5769-3045 } \\
& \text { Seunghyun Lee } \\
& \text { https://orcid.org/0000-0003-1858-0640 } \\
& \text { Yeon Jin Cho } \\
& \text { https://orcid.org/0000-0001-9820-3030 } \\
& \text { Jung-Eun Cheon } \\
& \text { https://orcid.org/0000-0003-1479-2064 } \\
& \text { In-0ne Kim } \\
& \text { https://orcid.org/0000-0001-5800-3536 } \\
& \text { Woo Sun Kim } \\
& \text { https://orcid.org/0000-0003-2184-1311 }
\end{aligned}
$$

\section{REFERENCES}

1. Kuroda S, Houkin K. Moyamoya disease: current concepts and future perspectives. Lancet Neurol 2008;7:1056-1066

2. Suzuki J, Kodama N. Moyamoya disease--a review. Stroke 1983;14:104-109

3. Research Committee on the Pathology and Treatment of Spontaneous Occlusion of the Circle of Willis, Health Labour Sciences Research Grant for Research on Measures for Infractable Diseases. Guidelines for diagnosis and treatment of moyamoya disease (spontaneous occlusion of the circle of Willis). Neurol Med Chir (Tokyo) 2012;52:245-266

4. Yun TJ, Cheon JE, Na DG, Kim WS, Kim IO, Chang KH, et al. Childhood moyamoya disease: quantitative evaluation of perfusion MR imaging--correlation with clinical outcome after revascularization surgery. Radiology 2009;251:216-223

5. Kim T, Oh CW, Bang JS, Kim JE, Cho WS. Moyamoya disease: treatment and outcomes. J Stroke 2016;18:21-30 
6. Matsushima T, Inoue T, Suzuki S0, Fujii K, Fukui M, Hasuo K. Surgical treatment of moyamoya disease in pediatric patients-comparison between the results of indirect and direct revascularization procedures. Neurosurgery 1992;31:401-405

7. Patel NN, Mangano FT, Klimo P Jr. Indirect revascularization techniques for treating moyamoya disease. Neurosurg Clin N Am 2010;21:553-563

8. Park JH, Yang SY, Chung YN, Kim JE, Kim SK, Han DH, et al. Modified encephaloduroarteriosynangiosis with bifrontal encephalogaleoperiosteal synangiosis for the treatment of pediatric moyamoya disease. Technical note. J Neurosurg 2007;106:237-242

9. Hasuo K, Tamura S, Kudo S, Uchino A, Carlos R, Matsushima T, et al. Moya moya disease: use of digital subtraction angiography in its diagnosis. Radiology 1985;157:107-111

10. Calamante F, Ganesan V, Kirkham FJ, Jan W, Chong WK, Gadian $D G$, et al. MR perfusion imaging in moyamoya syndrome: potential implications for clinical evaluation of occlusive cerebrovascular disease. Stroke 2001;32:2810-2816

11. Tibussek D, Rademacher C, Caspers J, Turowski B, Schaper J, Antoch G, et al. Gadolinium brain deposition after macrocyclic gadolinium administration: a pediatric case-control study. Radiology 2017;285:223-230

12. Mithal LB, Patel PS, Mithal D, Palac HL, Rozenfeld MN. Use of gadolinium-based magnetic resonance imaging contrast agents and awareness of brain gadolinium deposition among pediatric providers in North America. Pediatr Radiol 2017;47:657-664

13. Zhang J, Wang J, Geng D, Li Y, Song D, Gu Y. Whole-brain CT perfusion and $\mathrm{CT}$ angiography assessment of moyamoya disease before and after surgical revascularization: preliminary study with 256-slice CT. PLoS One 2013;8:e57595

14. Proisy M, Bruneau B, Rozel C, Tréguier C, Chouklati K, Riffaud $\mathrm{L}$, et al. Arterial spin labeling in clinical pediatric imaging. Diagn Interv Imaging 2016;97:151-158

15. Haller S, Zaharchuk G, Thomas DL, Lovblad K0, Barkhof F, Golay X. Arterial spin labeling perfusion of the brain: emerging clinical applications. Radiology 2016;281:337-356

16. Sugino T, Mikami T, Miyata K, Suzuki K, Houkin K, Mikuni $\mathrm{N}$. Arterial spin-labeling magnetic resonance imaging after revascularization of moyamoya disease. J Stroke Cerebrovasc Dis 2013;22:811-816

17. Zaharchuk G, Do HM, Marks MP, Rosenberg J, Moseley ME, Steinberg GK. Arterial spin-labeling MRI can identify the presence and intensity of collateral perfusion in patients with moyamoya disease. Stroke 2011;42:2485-2491

18. Hara S, Tanaka Y, Ueda Y, Hayashi S, Inaji M, Ishiwata K, et al. Noninvasive evaluation of CBF and perfusion delay of moyamoya disease using arterial spin-labeling MRI with multiple postlabeling delays: comparison with 150-Gas PET and DSC-MRI. AJNR Am J Neuroradiol 2017;38:696-702

19. Wang R, Yu S, Alger JR, Zuo Z, Chen J, Wang R, et al. Multidelay arterial spin labeling perfusion MRI in moyamoya disease--comparison with CT perfusion imaging. Eur Radiol
2014;24:1135-1144

20. Goetti R, O'Gorman R, Khan N, Kellenberger CJ, Scheer I. Arterial spin labelling MRI for assessment of cerebral perfusion in children with moyamoya disease: comparison with dynamic susceptibility contrast MRI. Neuroradiology 2013;55:639-647

21. Matsushima T, Fujiwara S, Nagata S, Fujii K, Fukui M, Kitamura $K$, et al. Surgical treatment for paediatric patients with moyamoya disease by indirect revascularization procedures (EDAS, EMS, EMAS). Acta Neurochir (Wien) 1989;98:135-140

22. Wu WC, Fernández-Seara M, Detre JA, Wehrli FW, Wang J. A theoretical and experimental investigation of the tagging efficiency of pseudocontinuous arterial spin labeling. Magn Reson Med 2007;58:1020-1027

23. Wang J, Zhang Y, Wolf RL, Roc AC, Alsop DC, Detre JA. Amplitude-modulated continuous arterial spin-labeling 3.0T perfusion MR imaging with a single coil: feasibility study. Radiology 2005;235:218-228

24. Wang Z, Aguirre GK, Rao H, Wang J, Fernández-Seara MA, Childress AR, et al. Empirical optimization of ASL data analysis using an ASL data processing toolbox: ASLtbx. Magn Reson Imaging 2008;26:261-269

25. Tatu L, Moulin T, Bogousslavsky J, Duvernoy H. Arterial territories of the human brain: cerebral hemispheres. Neurology 1998;50:1699-1708

26. Robertson RL, Burrows PE, Barnes PD, Robson CD, Poussaint TY, Scott RM. Angiographic changes after pial synangiosis in childhood moyamoya disease. AJNR Am J Neuroradiol 1997;18:837-845

27. Houkin K, Nakayama N, Kuroda S, Ishikawa T, Nonaka T. How does angiogenesis develop in pediatric moyamoya disease after surgery? A prospective study with MR angiography. Childs Nerv Syst 2004;20:734-741

28. Kim SK, Seol HJ, Cho BK, Hwang YS, Lee DS, Wang KC. Moyamoya disease among young patients: its aggressive clinical course and the role of active surgical treatment. Neurosurgery 2004;54:840-844; discussion 844-846

29. Yoon HK, Shin HJ, Lee M, Byun HS, Na DG, Han BK. MR angiography of moyamoya disease before and after encephal oduroarteriosynangiosis. AJR Am J Roentgenol 2000;174:195200

30. So Y, Lee HY, Kim SK, Lee JS, Wang KC, Cho BK, et al. Prediction of the clinical outcome of pediatric moyamoya disease with postoperative basal/acetazolamide stress brain perfusion SPECT after revascularization surgery. Stroke 2005;36:1485-1489

31. Ikezaki K, Matsushima T, Kuwabara Y, Suzuki S0, Nomura T, Fukui M. Cerebral circulation and oxygen metabolism in childhood moyamoya disease: a perioperative positron emission tomography study. J Neurosurg 1994;81:843-850

32. Saida T, Masumoto T, Nakai Y, Shiigai M, Matsumura A, Minami M. Moyamoya disease: evaluation of postoperative revascularization using multiphase selective arterial spin labeling MRI. J Comput Assist Tomogr 2012;36:143-149 
33. Yun TJ, Paeng JC, Sohn CH, Kim JE, Kang HS, Yoon BW, et al. Monitoring cerebrovascular reactivity through the use of arterial spin labeling in patients with moyamoya disease. Radiology 2016;278:205-213

34. Goetti R, Warnock G, Kuhn FP, Guggenberger R, O'Gorman $R$, Buck $A$, et al. Quantitative cerebral perfusion imaging in children and young adults with moyamoya disease: comparison of arterial spin-labeling-MRI and H2[150]-PET. AJNR Am J Neuroradiol 2014;35:1022-1028

35. Blauwblomme $T$, Lemaitre $H$, Naggara 0 , Calmon R, Kossorotoff $M$, Bourgeois $M$, et al. Cerebral blood flow improvement after indirect revascularization for pediatric moyamoya disease: a statistical analysis of arterial spin-labeling MRI. AJNR Am J Neuroradiol 2016;37:706-712

36. Lee S, Yun TJ, Yoo RE, Yoon BW, Kang KM, Choi SH, et al. Monitoring cerebral perfusion changes after revascularization in patients with moyamoya disease by using arterial spin- labeling MR imaging. Radiology 2018;288:565-572

37. Lee M, Zaharchuk G, Guzman R, Achrol A, Bell-Stephens T, Steinberg GK. Quantitative hemodynamic studies in moyamoya disease: a review. Neurosurg Focus 2009;26:E5

38. Roach BA, Donahue MJ, Davis LT, Faraco CC, Arteaga D, Chen $\mathrm{SC}$, et al. Interrogating the functional correlates of collateralization in patients with intracranial stenosis using multimodal hemodynamic imaging. AJNR Am J Neuroradiol 2016;37:1132-1138

39. Yoo RE, Yun TJ, Rhim JH, Yoon BW, Kang KM, Choi SH, et al. Bright vessel appearance on arterial spin labeling MRI for localizing arterial occlusion in acute ischemic stroke. Stroke 2015;46:564-567

40. Harreld JH, Helton KJ, Kaddoum RN, Reddick WE, Li Y, Glass J0, et al. The effects of propofol on cerebral perfusion MRI in children. Neuroradiology 2013;55:1049-1056 